\title{
PERCEPTIONS OF PHYSICIANS, MEDICAL AND NURSING STUDENTS CONCERNING SHARED DECISION-MAKING:
}

\section{A CROSS-SECTIONAL STUDY}

\section{Background}

- Shared decision-making (SDM) is an essential factor in attaining a patient-centered health care.

- The process of integrating

$\checkmark$ Best available evidence

$\checkmark$ Knowledge, preferences and values of patient and provider

- Benefits of implementation such as

$\checkmark$ Improvement of patient-relevant outcomes

$\checkmark$ Decrease in costs

$\checkmark$ Reduction of health inequalities

$\checkmark$ Empowerment of patients

- Lagging adoption at level of health care providers.

- Research regarding the attitudes of providers and students in various countries is scarce.

- Main objective to evaluate and compare the sharing attitudes of physicians, medical and nursing students in Flanders (Belgium).

\section{Methods}

A cross sectional study was conducted between June through September 2017.

- Patient-Practitioner Orientation Scale (PPOS)

$\checkmark$ Six-point Likert scale

$\checkmark$ Forward translation English -> Dutch

$\checkmark$ Known groups validation

$\checkmark$ Sharing subscale $(\alpha 0.70)$

$\checkmark$ Demographic questionnaire

- Inclusion criteria

$\checkmark$ Third year Master of Science in Medicine

$\checkmark$ Master of Science in Nursing

$\checkmark$ Physicians

- Data analysis

$\checkmark$ Independent t-tests

$\checkmark$ One and two way analysis of variance

$\checkmark$ Multivariable linear regression model

\section{Results}

- Total of 266 respondents

$\checkmark$ Physicians ( $n=93 ; 4,24 \pm 0,64)$

$\checkmark$ Medical students $(n=147 ; 4,30 \pm 0,61)$

$\checkmark$ Nursing students $(n=26 ; 4,30 \pm 0,67)$

- Multivariable regression

$\checkmark$ Female sex $(p<0,10)$ and working in general practice or internal medicine $(p<0,05)$ predictive for higher sharing among physicians

- Difference $(p<0,05)$ based on physicians' specialism

$\checkmark$ Paediatrics $(4,79 \pm 0,69)$

$\checkmark$ Psychiatry $(4,74 \pm 0,47)$

$\checkmark$ Gynaecology/Obstetrics $(4,40 \pm 0,38)$

$\checkmark$ General Practice $(4,31 \pm 0,59)$

$\rightarrow$ Significantly higher than Surgery $(3,84 \pm 0,58)$

- No difference between occupation, age, rank, preferred future specialisation.

\section{Conclusion}

Health care providers and students in Flanders are generally less sharing than samples from the US.

- Strong perception that the physician should decide and that the patient must rely on their knowledge.

- Important influence of culture on decision making.

- Sharing attitudes presumably decrease or increase due to exposure to the clinical context.

- Urgent need for health policy and educational institutions to facilitate a supporting environment. 